\title{
Caching on Named Data Network: a Survey and Future Research
}

\author{
Leanna Vidya Yovita, Nana Rachmana Syambas
}

School of Electrical Engineering and Informatics, Bandung Institute of Technology, Indonesia

\begin{tabular}{l} 
Article Info \\
\hline Article history: \\
Received Mar 20, 2018 \\
Revised Jul 16, 2018 \\
Accepted Jul 30, 2018 \\
\hline Keyword: \\
Caching placement \\
Caching policy \\
Content selection \\
Content storage \\
Named data network
\end{tabular}

\begin{abstract}
The IP-based system cause inefficient content delivery process. This inefficiency was attempted to be solved with the Content Distribution Network. A replica server is located in a particular location, usually on the edge router that is closest to the user. The user's request will be served from that replica server. However, caching on Content Distribution Network is inflexible. This system is difficult to support mobility and conditions of dynamic content demand from consumers. We need to shift the paradigm to content-centric. In Named Data Network, data can be placed on the content store on routersthat are closest to the consumer. Caching on Named Data Network must be able to store content dynamically. It should be selectively select content that is eligible to be stored or deleted from the content storage based on certain considerations, e.g. the popularity of content in the local area. This survey paper explains the development of caching techniques on Named Data Network that are classified into main points. The brief explanation of advantages and disadvantages are presented to make it easy to understand. Finally, proposed the open challenge related to the caching mechanism to improve NDN performance.
\end{abstract}

Copyright $\odot 2018$ Institute of Advanced Engineering and Science. All rights reserved.

\section{Corresponding Author:}

Leanna Vidya Yovita,

School of Electrical Engineering and Informatics,

Bandung Institute of Technology,

10 Ganesha Road, Lb. Siliwangi, Coblong, Bandung 40132, West Java, Indonesia.

Email: leanna@ telkomuniversity.ac.id

\section{INTRODUCTION}

The era of telecommunications began in 1876, where a network was built that enabled 2 parties to transmit their voice and communicate.The Internet began in 1969s, funded by Advanced Research Project Agency [1]. Using Internet Protocol (IP) as an address, the request from the user will be forwarded to the server, through other nodes within the network. The replies tothe request will be sent to the user through a particular path that has been formed by routing process in the network. If any user requests the same data, then the packet will be sent again from the server to the user. This causes inefficient packet delivery process because the packet is always sent from a server that is far from the user. To solve this problem, the concept of Content Distribution Network [2] was proposed. A replica server is created contains all the data as in the main server, placed at a fixed location, closer to the user. So that, the request for certain content will be redirected to the replica server and it is no need to be served by an origin server that is farther away.

The replica server is updatedperiodically or when any content changes on its original server. However, this system will be difficult to support mobility and dynamic changing content request from consumers. When the consumer away from the replica server, it leads to the possibility that a consumer can no longer be served efficiently by the replica server. Content Distribution Network that is still based on Internet Protocol (IP) causes the request process from the user is always addressed to a particular server. Consequently, anadditional process is still needed tomapping the intended IP with the server position that is 
closest to the user. Actually, from the beginning, the focus of the user request is the content (content-based), but in the previous system, the request is addressed to a certain server node with a certain IP (host-based).

In 2009, Jacobson et al. propose a content-based network paradigm [3]. This concept has been raised a few years earlier in its research projects and it is named Content-Centric Networking (CCN) originally developed at Xerox's Palo Alto Research Center. It is currently developing into Named Data Network (NDN) initiated by the NSF-Funded Future Internet Architecture Project [4]. This concept replaces the 'where' paradigm to the concept of'what', where the consumer request is no longer addressed to a specific node but it is intended for a certain content [3], [5]-[7]. This paradigm causes the response to the content requests not only served by a particular server but also can be served by the nearest device which stores the requested data. To support this concept, the NDN router nodes are equipped with content storage to store the data [3]-[6], [8].

The concept of caching on Named Data Network is different from caching in the previous system. Each NDN node has a content storage to hold data. Different with the previous network, node mobility will be supported because the content store can be tailored to the user's demand pattern for the content. Changes in user positions cause the router has to re-customize the contents in the content storeaccording to the user requests in the local area. Cachein NDN is more dynamic. The NDN architecture supports flexible network topologies, where wireless nodes can enter and exit the area. One time, a node can be a producer and sometimes it turns into theconsumer. In the NDN node can be embedded with various cache rules, including to determine which content will be selected and deleted from content store [5], [9], [10], the selection of places where a content will be cached [10]-[13], and cache policy that imply the model of cooperation between nodes to determinethe caching decision [14]-[17]. Related to node mobility, several techniques have been studied to maintain the performance of the system even though the nodes move in and out of coverage [18]-[21].

This survey paper explains the development of caching techniques on Named Data Network which is an important basis for understanding the latest NDN caching techniques in developing better future techniques for enhancing NDN performance as an efficient forward communication solution. The brief explanation of advantages and disadvantages are presented to make it easy to understand. Finally, proposed the open challenge related to the caching mechanism to improve NDN performance.

The remainder of this paper is organized as follow. In section 2 described the state of the art of caching on the Named Data Network. The caching techniques are grouped into cache placement, cache content selection, and cache policy design. In this sectionalso explained the advantages and drawbacks of each group of caching techniques. The technique to support mobility are explained too. In section 3 described the challenge and open issues related to the caching in Named Data Network. And finally, section 4 put forward the conclusion of this paper.

\section{RELATED WORK}

Some survey papers on caching have been done before. Paper [22] emphasizes discussion on techniques of cache replacement for web services in theIP-based system. Paper [23] emphasizes discussion on several in-network caching mechanisms in Information-Centric Networking, 2014 and earlier. Paper [5] discusses information-centric mobile caching, including caching in cellular, ad hoc, and ahybrid network. This paper explains different cache location for each scheme and some cache mechanisms. Discussion on caching replacement mechanism only, presented by paper [22] and [24], for themobile node. Due to the author's knowledge, so far there has been no paper survey that discusses the latest caching techniques and mapping the technique in groups based on the basic technique. This scheme makes it easy to understand the basic techniques of the caching mechanisms.

This survey paper focuses on caching techniques, including the recent studies. To make it clear to explain, in this paper the caching mechanism is divided into 4 general group, they are cache placement, cache content selection, cache policy design andcaching for mobile nodes. The explanation begins with the caching differences in Named Data Network with its previous network, the urgency of caching discussion on Named Data Network to improve its performance, and then mapping the caching techniques based on the basic mechanism. The aim is to facilitate the reader to know the basis of the development of these caching techniques. The advantages and disadvantages of each group of techniques are presented with a succinct and focus to make it easy to understand. This paper concludes with an explanation of the proposed research on caching on NDN that is still open, so it can continue to be developed to examine the best techniques to support NDN. 


\section{STATE OF THE ART OF CACHING ON NAMED DATA NETWORK}

\subsection{Component of Named Data Network Router}

Named Data Network shifts the 'where' to 'what' paradigm. The user sends his request for a content to the network, and then the network woulddetermines who is the most efficient node that can serve this request. So, the user does not need to know where is the content server. This paradigm causes the reply for a request not always be done by the server, but any node that is in the network. The NDN architecture causes data communication processes more efficient and network loads will be significantly reduced.

The NDN Node consists of 3 components, namely Content Storage (CS), Pending Interest Table (PIT) and Forwarding Information Based (FIB) [3], [25]. When consumer B wants a data from the producer, the consumer will send a request for certain content using the Interest Packet. The NDN router that receives the request from the consumer will check whether the content is in its CS. If there is, the router will immediately send the requested data to the consumer. If the data is not in CS, then the router checks the Pending Interest Table to see if the content has been requested and has not been replied with matching data packets. If in the PIT there is such information, then the information will be updated by adding information that consumer B also requested the same data. The information on this PIT makes a reverse path for sending data to the consumer. If in the PIT there is no data request content that is the same as consumer B, then checks are made on Forwarding Information Based (FIB). The interest packet will be forwarded to the data provider node according to the information in FIB. If FIB does not store the content provider's node data, the interest packet will be discarded by the NDN router. This process described in Figure 1.

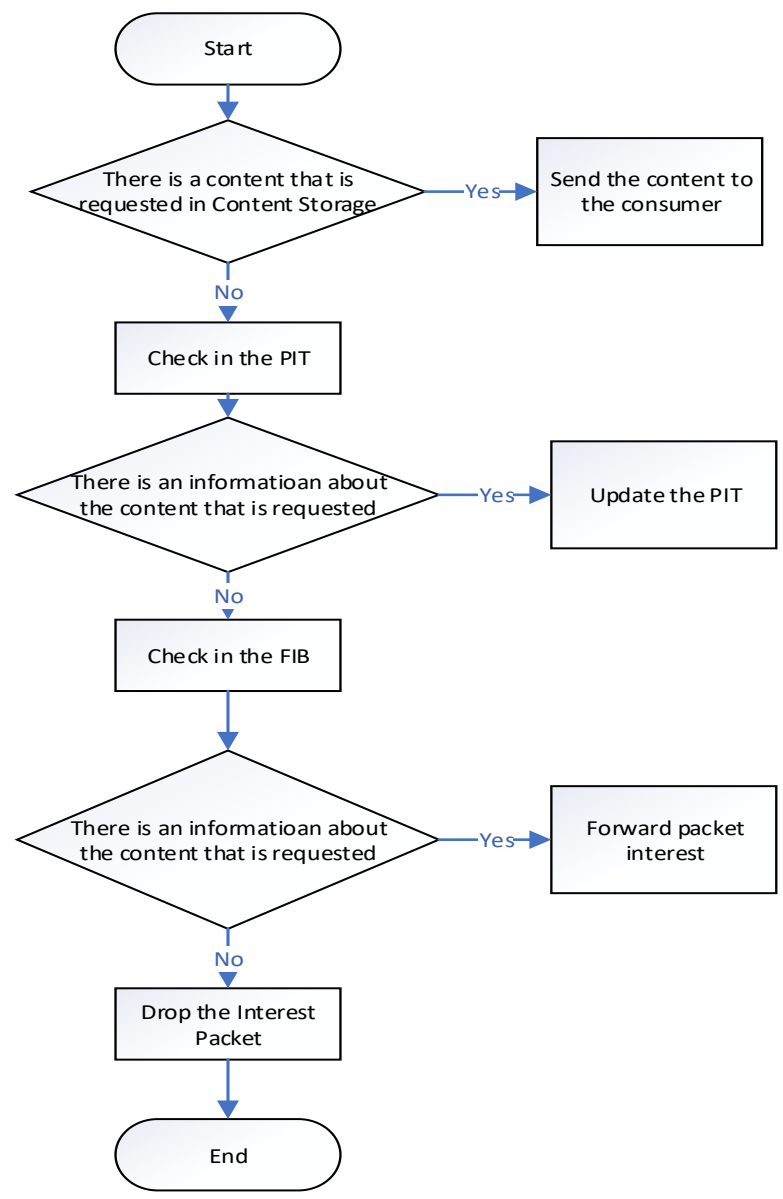

Figure 1. Processes that occur on each NDN router when it receives the Interest Packet [25]

\subsection{Content Storage}

Content Storage (CS) is one of the important components in the NDN router node. CS is essential to allow the data to be stored in NDN router nodes so that if the consumer request for a content, it is not necessarily served by the certain server, but can be served by a router node that has the content in its CS. CS 
is one of the limited resources on NDN routers. Therefore it should be utilized as efficiently as possible in order to improve NDN performance.

The size of the content store affects the delay and number of hops that packets must take to go to consumer [26]. This condition affects the overall network load due to the circulation of data in the network [4], [8]. CS also performs different effect with the various cache policy implemented in the node [27]. In this paper, the caching strategies are classified as cache placement, cache content selection, cache policy design and caching for themobile node.Each group described, including its advantages and drawbacks in sections 3.3 to 3.6 .

\subsection{Cache Placement}

Cache placement focuses on determining which nodes will store a data packet. In the Publisher/Subscriber network, it has been proposed a methodto choose a node to store packets based on local content popularity and content storage capacity per node [28]. In NDN networks, packets are initially placed on every node in the network so that the consumer can directly access the content to the closestnode.

Paper [11] proposes a packet data flooding mechanism, where data packets are stored in all nodes that are in the best path but limited to the maximum number of hops for the spread of the packet. In paper [12] the package is deployed to be stored in network nodes but still maintained to ensure there are no redundant packets, to save resources, using bloom filter. The lack of bloom filter technique relatedto false positive problems corrected by A. Hidayat et al [13]. In this technique, bloom filter is combining with sequential search algorithm.

Paper [29] proposes a mechanism that combines the technique of packet insertion and packet deletion by adding a Caching Contribution parameter in the interest packet. The node will decide whetherit will cache the data packet or not. If the data packet cannot be cached on the certain node, then it will be forwarded to another node. A trail mechanism is built to stores information about the path to the next node that can store the content.In paper [10], nodes that often get a content request from consumer have a high contribution value. A node will store a content that has high contribution value if storage capacity is available. Paper [9] proposes the movement of data toward the edge router closest to the consumer for every specific content request. The cache placement can be resume into 3, i.e. function based, diversity and flooding as shown in Figure 2. Comparison of the three techniques, including the technical focus, the advantages, and drawbacks described in Table 1.

Table 1. Comparison of Cache Placement Techniques

\begin{tabular}{|c|c|c|c|}
\hline Classification & Main focus & Advantages & Drawbacks \\
\hline $\begin{array}{l}\text { Function- } \\
\text { based } \\
{[28][10][29]}\end{array}$ & $\begin{array}{l}\text { Focus on specific parameters to } \\
\text { be achieved. i.e. a minimum } \\
\text { delay, the number of hops, etc. }\end{array}$ & $\begin{array}{l}\text { Specifically to maximize } \\
\text { the achievement of } \\
\text { certain parameters }\end{array}$ & $\begin{array}{l}\text { - The algorithm is more complex if } \\
\text { there are many parameters. }\end{array}$ \\
\hline $\begin{array}{l}\text { Diversity } \\
\text { [9] [12] }\end{array}$ & $\begin{array}{l}\text { Emphasize the spread of } \\
\text { content on the network by } \\
\text { avoidingto keep the same } \\
\text { packets in the network. to save } \\
\text { resources on the network }\end{array}$ & $\begin{array}{l}\text { More efficiently store } \\
\text { content because only } \\
\text { different contents that are } \\
\text { stored by different nodes }\end{array}$ & $\begin{array}{l}\text { - } \quad \text { Larger average delay } \\
\text { Have to cooperate with other } \\
\text { nodes in implementing content } \\
\text { storage rules } \\
\text { - The processing time may increase } \\
\text { as it involves knowledge from } \\
\text { other nodes }\end{array}$ \\
\hline $\begin{array}{l}\text { Flooding } \\
\text { [3] [11] }\end{array}$ & $\begin{array}{l}\text { Emphasize to store content as } \\
\text { much as possible on the } \\
\text { network to ensure the ease and } \\
\text { speed of consumers in } \\
\text { accessing data }\end{array}$ & $\begin{array}{l}\text { - Users can access } \\
\text { packages with minimal } \\
\text { delay } \\
\text { - } \\
\text { Requests for content can } \\
\text { be served by multiple } \\
\text { nodes }\end{array}$ & $\begin{array}{l}\text { - Network loads increase because } \\
\text { nodes store the same content. }\end{array}$ \\
\hline
\end{tabular}

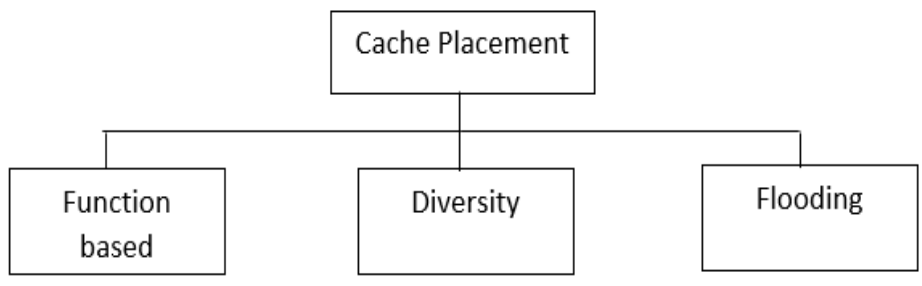

Figure 2. Classification of cache placement techniques 


\subsection{Cache Content Selection}

The cache content selection techniques focus on determining which content will be cached and which content should be removed from the cache. Some of the content selection techniques to cache are Caching Everything Everywhere (CEE) [3], [23], where each node stores all of the data from the producer and it means no content selection and Prob (p) [3], [5], [23] where data is cached with probability p and not cached with probability 1-p. As a result, data packets that are cached by one router may be different from the other routers. Paper [30] proposes the concept that every router cache the data with the probability determined by the number of hop between producer and the router.Selection of content to be cached based on the prediction that the content will be requested by the local consumer proposed by paper [31]. Related to the cache content selection, content centric network performance is also affected by CS replacement rules and user localization [32]. The cache content selection can be resume as in Table 2.

Table 2. Comparison of Cache Content Selection Techniques (Insertion and Eviction)

\begin{tabular}{|c|c|c|c|}
\hline Classification & Main focus & advantages & Drawbacks \\
\hline $\begin{array}{l}\text { Popularity } \\
\text { [29] [8] [36] }\end{array}$ & $\begin{array}{l}\text { Focus on packet } \\
\text { selection based on the } \\
\text { number of requests for } \\
\text { the packet. }\end{array}$ & $\begin{array}{l}\text { Its already accommodated the } \\
\text { selection of content based on } \\
\text { consumer interest }\end{array}$ & $\begin{array}{l}\text { less popular content can be omitted, while it is } \\
\text { still needed or requested by some consumers }\end{array}$ \\
\hline $\begin{array}{l}\text { Probability } \\
\text { [3] [5] [23] } \\
\text { [37] }\end{array}$ & $\begin{array}{l}\text { Emphasize the selection } \\
\text { of packages/content } \\
\text { with a certain } \\
\text { probability. A packet } \\
\text { can be cached with a } \\
\text { certain probability. }\end{array}$ & $\begin{array}{l}\text { More fair in determining the } \\
\text { package to be cached or deleted. }\end{array}$ & $\begin{array}{l}\text { - } \quad \text { Some nodes may store the same content } \\
\text { - Need specific strategies to determine the } \\
\text { probability }\end{array}$ \\
\hline $\begin{array}{l}\text { Predic- } \\
\text { tionbased } \\
{[34][31]}\end{array}$ & $\begin{array}{l}\text { Tighten the selection of } \\
\text { packages based on } \\
\text { predictions whether the } \\
\text { selection of content will } \\
\text { provide the target value } \\
\text { set. }\end{array}$ & $\begin{array}{l}\text { - Avoidstoring unnecessary } \\
\text { content } \\
\text { - Accommodate the future } \\
\text { needs of the user }\end{array}$ & $\begin{array}{l}\text { - The prediction may be incorrect if the } \\
\text { condition of the network or user changes. } \\
\text { - Internal calculation of the router is more } \\
\text { complex. }\end{array}$ \\
\hline
\end{tabular}

Another technique related to the cache content selection is Prediction-based caching [33]. The content will be decided to be cached by router based on the number of requests. In this scheme, it is added a new table in the router, named the Pending Species Interest Table (PSIT). This table stores the list of the most requested content based on data in the PIT. Suppose there is content that is regularly requested by the consumer every Monday, but there is also non-regular content, for example, the contents of the World Cup event. After that, Dynamic Cache Adjustment algorithm is used to decide a package that will be cached or not based on its wastage value. A content will be viewed in size. If the CS is still sufficient, the package is stored. If the CS is full then the packet in the CS will be select randomly and then compared it with the new data packets. If they are both same, the value of the hit parameter will increase. Re-testing is done by comparing the hit parameter with the amount of data that has been sorted. If the hit value is higher, the packet is given allocation in the buffer, and otherwise, the content is not allocated in CS. Selection of a content can also be calculated based on local popularity and hop count reduction gain that can be given by the packet [29].

Another content selection technique is Max-Gain In-network Caching (MAGIC) [34]. The proposed method aims to reduce bandwidth consumption and consider content popularity as well as hop reduction. When receiving the interest packet, each router will calculate the Local Gain and compare it with the value stored on the MaxGain field. If the local cache of the router gain is greater than the MaxGain value, then the router will update the MaxGain value in the interest packet. This MaxGain value will be copied on additional fields in the data packet. Along the packet delivery path, if the Local Gain value is the same as the MaxGain value in the data packet, it will be cached in the data packet.

If a data packet enters the router node and the router didn't have it in its Content Storage, then the node will check its Content Storage condition. If it is full, then it will be selected which packet will be deleted from Content storage to provide space to store the new packet. Techniques that are commonly used in the NDN system to select which packets will be deleted in CS is Least Recently Used (LRU) and Least Frequently Used (LFU) [3], [29], [22]. Deghgan et al in the paper [2] proposed another technique to give a timer to a package. The timer is used to determine how long a packet may be in the content storage before it is finally deleted. Paper [35] proposed the Recent Usage Frequency (RFU) algorithm, which determined the popularity of content within a limited time range. The lowest popularity value will cause a content to be removed from the content store. 
According to the paper [24], the performance of caching can be improved by using efficient caching replacement methods. In mobile networks, this is a challenge, becausethe environment is different from the fixed network conditions. The parameters used by the replacement rule include recency, popularity, message size, cost to achieve objects, and access delay [24]. The cache content selection techniques can be resume as in Figure 3 and the comparison of cache content selection techniquesas in Table 2.

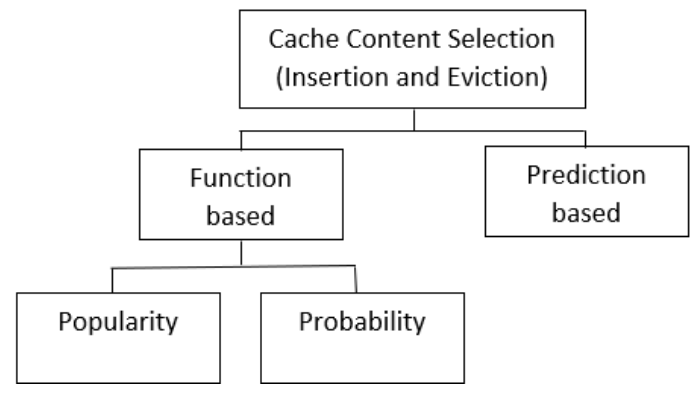

Figure 3. Classification of cache content selection techniques

\subsection{Cache Policy Design}

Cache policy focuses on techniques how content is stored in nodes. One of the cache policy related techniques is Utility-driven caching [8]. This technique is a utility-driven caching technique in which a utility value is linked to a content. Utilities are a function of a hit possibility of content. The goal is to maximize the total amount of utility content in content storage.

Paper [38] modeled the cache on its system into 2 layers. The first layer is the individual caching in each node and layer 2 is the accumulation of all the cache on the network. The study analyzes how much storage content should be provided in the system to meet the performance of 4 applications, i.e. web traffic, file sharing, and video traffic that are distinguished into user-generated content (UGC) and video on demand $(\mathrm{VoD})$.

Assantachai et al [14] proposed a hybrid caching scheme. If any new content is requested by the consumer and not exist yet on the router node, then the new content will be saved. The content replacement scheme used is a combination of the concept of a cooperative approach and distributive approach. Cooperative caching is a scheme in which each node makes a replacement decision based on the knowledge received from other nodes residing in the same region. Distributive caching is used to make decisions independently using internal knowledge to achieve local maximum performance. In paper [14] the network is divided into 2 parts, that is the normal region (region on the edge) and the backbone region (the region that connects the normal regions). In the normal region, if there is a cache hit interest, the content is moved to the front of the sequence, and when the cache misses then the data at the tail of the sequence is removed. The backbone region follows the normal region pattern, only the backbone nodes work with other nodes in the same region to decide to cache. Cooperative caching policy design is also used in [39] with areas divided into clusters

Paper [15], [40] described that the mechanism to cache a content has a crucial impact on the efficiency of content delivery and utilization of CS. Paper [9] proposes the mechanism to divide files into smaller packets called chunk. The amount of chunk disseminated depends on the popularity of the content. The number of chunks is determined by the Chunk Marking Window (CMW) which exponentially enlarges every number of chunks successfully delivered

In [41] Content-Centric network is implemented using two types of applications. For each application, it is created a separate list and each identified with a unique ID. The CS is separated and each application can only be stored in its own content store. The storage content partition mechanism is tested with two methods: static cache partitioning and dynamic cache partitioning. In static partitioning, the cache can only be used as specified. While in dynamic cache partitioning, unused cache by an application can be shared with other applications. Cache with splitting technique also proposed in [42]. The content storage is divided into two part, one part for a popular content and the other for less popular content. Paper [43] split the content storage into three regions. The data is categories as a self-data, friends data, and stranger data. Paper [16] more specific on caching management in memory where multiprocessor is used with certain interconnect mechanisms to reduce power usage.

Caching techniques that coupling data cache placement, replacement, and location was proposed by Xiaoyan $\mathrm{Hu}$, et al. [29]. To set the packet to be cached, it is defined a caching value for each packet o that 
can be cached at node $\mathrm{v}$. This caching value involves multiplication of local popularity value and hop count reduction gain of the item, then divided by cache space contention which is the same value in all routers. If an interest goes to node $\mathrm{v}$, and the item wasnot cached yet on the node $\mathrm{v}$ before, node $\mathrm{v}$ will calculate the approximate potential value of the caching contribution of the item. The data will be cached at node $\mathrm{v}$ if the maximal value of caching contribution is positive. If the content storage is full, it will select a package with the least contribution caching value to delete. Related to the caching location determination, the cache location component will maintain the trail to guide the content. This trail is only created if the content is not cached on the local node. The cache policy design can be resume as in Figure 4. The comparison of cache policy design techniques as in Table 3 .

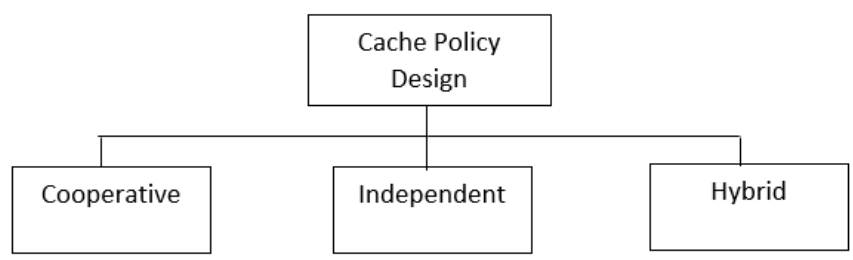

Figure 4. Classification of cache policy design

Table 3. Comparison of cache policy design techniques

\begin{tabular}{|c|c|c|c|}
\hline Classification & Main focus & Advantages & Drawbacks \\
\hline $\begin{array}{l}\text { Cooperative } \\
{[9][29][44]} \\
{[17]}\end{array}$ & $\begin{array}{l}\text { Coordinate and collaborate with } \\
\text { other nodes in the network to } \\
\text { determine caching policy }\end{array}$ & $\begin{array}{l}\text { More efficient in using } \\
\text { resources }\end{array}$ & 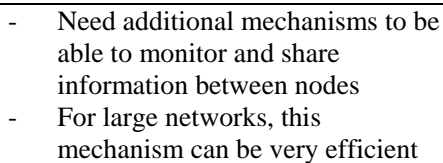 \\
\hline $\begin{array}{l}\text { Independent } \\
{[8][31][42]} \\
{[38][45][46]}\end{array}$ & $\begin{array}{l}\text { Caching-related decisions are } \\
\text { performed by the node regardless of } \\
\text { information from other nodes }\end{array}$ & $\begin{array}{l}\text { There is no need for } \\
\text { additional mechanisms } \\
\text { for monitoring and } \\
\text { sharing information with } \\
\text { other nodes }\end{array}$ & - Can not do resource sharing \\
\hline $\begin{array}{l}\text { Hybrid } \\
{[14]}\end{array}$ & $\begin{array}{l}\text { Merging between cooperative and } \\
\text { independent techniques. }\end{array}$ & $\begin{array}{l}\text { - Can more efficiently } \\
\text { apply certain mechanis } \\
\text { ms to specific conditions }\end{array}$ & $\begin{array}{l}\text { - Need to be defined about the } \\
\text { specific conditions for a } \\
\text { mechanism } \\
\text { - } \quad \text { Add the computation process }\end{array}$ \\
\hline
\end{tabular}

\subsection{Caching for Themobile Node}

Generally, caching techniques for mobile nodes have a basic idea for subscribing a user to a content producer [28], [47], prefetching content to other router that will handle consumer [19], [20], collaborate the data transmission mode for VANET [44], and mobile node support techniques that consider an energy [48]. In the mobile environment, the problems are NDN nodes always move, including routers, producers, and consumers [21]. The producer movement causes a greater problem than the movement of the consumer node or router node. Problem-solving related to producer movements is presented by paper [28].

The publish/subscribe system is the mechanism by which the subscriber can receive messages from the publisher. This relationship is governed by the manager so auser who subscribes to certain content will always get the content they want when publisher generates the content [47]. In the pre-existing pub/subsystem, theproducer does not store messages that have been published before. In this case, if new subscribers join the system, they could not get the content that has been published before theyenter the system. To solve the problem, [28] proposed storage mechanism and replication algorithm with differentiated content class. In this new system, storage can convert the content classes they store. The proposed replication algorithm is to select $\mathrm{M}$ storage points from $\mathrm{N}$ points that are available in the network based on locality and popularity, target replication degree of each topic, and storage capacity

A technique for accommodating consumer mobility in wireless networks is Proactive Multilevel Cache Selection (PMCS), proposed by paper [18]. In this scheme, if the consumer will switch coverage or handoff, the consumer will send a notification about which router to go to. The currently used router will select a subset of neighboring routers to receive content that has been requested by the consumer but has not yet been sent to it. When a handoff occurs, the consumer will stop requesting to send data. During this handoff process also, the destination router will cache the data packets from the old router, which has not 
been received by the consumer up to a certain limit. Once the connection to the new router has been established, then the data transmission will be served by the new router. Another technique is proposed by [19] to predict node mobility and provide the best prefetching node.

Paper [20] explain the mechanism to support producer mobility, such as push to send the data, make some copies of data, determine the content placement, and re-announce if they move to another area. Paper [44] propose VANET's communication mode switching, Vehicle-to-vehicle (V2V) and vehicle-toinfrastructure (V2I), depending on the popularity of downloaded content. Mobile node has the limited power, so the caching process has to consider the energy consumption in the node, due to green NDN as explained in [49]. Paper [48] proposed an energy efficient techniques for MANET. The network is divided into groups, managed by a Master Node. Paper [50] proposed a technique with optimal selection of cluster head in Wireless Sensor Network to improve efficiency.

\section{CHALLENGE AND OPEN ISSUES}

\subsection{QoS-based Caching}

In all caching techniques, either cache placement, cache content selection, or cache policy design that has been developed mostly have not considered the different treatment for different services. In studies that have been done, the data usually only differentiated based on content popularity, content recently, the estimated benefits of content storage, etc. There are only afewof studies that take into account the treatment differentiation based on service requirements or user requirements. In fact, different users may subscribe to different privileged services. So far, not much research has been done related to QoS-based caching on NDN. Paper [45] is one of the papers that discuss this distinction using classes.

The concept follows the Differentiated Service (DiffServ) concept that was previously used in the IP network. Further development is needed for caching mechanisms that can meet different requirements for services and users. These techniques include how to choose content and where tocache them in the network. The decision can be taken independently or cooperatively with other nodes in the network.

\subsection{Caching for Mobile Node}

Node mobility must be supported to provide the flexibility of the system. Generally, mobility characteristic is divided into producer mobility dan consumer mobility. Router mobility is similar to the consumer mobility. Consumer mobility is naturally supported by NDN, but not so with producer mobility. So, the area of the producer-mobility support technique is one of research opportunities. Several techniques are presented related to cache in the mobile node to support producer mobility. For Example, in the paper [18] pre-fetching content is proposed. This scheme was done when the mobile node moves to the new coverage router. Another proposed method is to prefetch a group of content, not just 1 content, which is usually requested by the consumer [31]. Pre-fetching causes additional time needed to move content to a new router. Further investigation of other techniques related to node mobility support for NDN is required to ensureuninterrupted data communicationseven if the user switches coverage by considering the expected delay, cache load, and the complexity of the algorithm that must be executed.

\subsection{Energy-aware Caching}

NDN routers in themobile wireless network will have power restrictions. Caching techniques that consider the availability of power on the node also need to be explored further. This process may include selecting nodes to place content based on position, distance, energy availability at the node, resource availability and other important things that should consider process efficiency. Covering a technique that can reduce the number of replacements that occur. If a content is too frequently removed from the cache, it will not be efficient.

\subsection{Type of Data on Content Store}

Currently, the cached content on the NDN router can be either a file or smaller, called chunk [9] [44]. Chunk-based systems will make the transmission process more efficient because if a chunk is lost during transmission or it is deleted in CS, it only needs to be replaced with a new chunk without having to replace the whole file. However, the division of the file into chunk causes the user's queries to be generated chunk-based. This means that in the chunk-based system, the interest packet for a complete file more than the file-based system. Further exploration of caching procedures and mechanisms regarding this form of data should be explored. 


\section{CONCLUSION}

In this paper, we have explicate the advantages of NDN network architecture compared to traditional IP network and Content Distribution Network, and excess caching on NDN compared to its predecessor system. The development of various caching techniques has been mapped out.In this paperalso explained the advantages and drawbacks of each group. Finally, it has been suggested the research opportunities related to caching on NDN that can be investigated in the future, i.e. caching mechanisms that involve differences in QoS requirements for data and users, caching that supports mobility nodes, and caching that considers energy.

\section{ACKNOWLEDGMENT}

This work was supported by Telkom University and Ministry of Research Technology and Higher Education Republic of Indonesia.

\section{REFERENCES}

[1] T.M. Chen, "From Circuit Switched to IP-based Networks", in Encyclopedia of Multimedia Technology and Networking, 2nd ed., M. Pagan, Ed. Idea Group Publishing, 2009.

[2] B.G. Pallis and A. Vakali, "Insight and Perspectives for Content Delivery Networks", vol. 49, no. 1, pp. 101-106, 2006.

[3] V. Jacobson, D.K. Smetters, N.H. Briggs, J.D. Thornton, M.F. Plass, and R.L. Braynard, "Networking Named Content", in The 5th ACM International Conference on emerging Networking Experiments and Technologies, 2009, pp. $1-12$.

[4] L.V. Yovita and N.R. Syambas, "Content Storage Effect on the Named Data Network Traffic Load", in The International Conference on Telecommunication Systems, Services, and Applications 2017, 2017.

[5] H. Jin, D. Xu, C. Zhao, and D. Liang, "Information-centric mobile caching network frameworks and caching optimization : a survey", EURASIP J. Wirel. Commun. Netw., vol. 33, pp. 1-32, 2017.

[6] Anjali, "Components of Named Data Networking”, Int. J. Innov. Eng. Technol., vol. 7, no. 3, pp. 543-552, 2016.

[7] Y. Jing, "Evaluating Caching Mechanisms In Future Internet Architectures", Cambridge, USA, MIT-CSAIL-TR2016-009, 2016.

[8] M. Dehghan, L. Massoulie, D. Towsley, D. Menasche, and Y.C. Tay, "A Utility Optimization Approach to Network Cache Design", in IEEE INFOCOM 2016, 2016.

[9] K. Cho, M. Lee, K. Park, T.T. Kwon, and Y. Choi, "WAVE: Popularity-based and Collaborative In-network Caching for Content-Oriented Networks", in 2012 IEEE Conference on Computer Communications Workshops, 2012, pp. 316-321.

[10] D. De Voluceau, L.C.C, and F. Sailhan, "Energy-aware Web Caching for Mobile Terminals", in The 22 nd International Conference on Distributed Computing Systems Workshops, 2002, pp. 820-825.

[11] C.M.I.N. Park and R.A. Rehman, "Packet Flooding Mitigation in CCN-based Wireless Multimedia Sensor Networks for Smart Cities Packet Flooding Mitigation in CCN-Based Wireless Multimedia Sensor Networks for Smart Cities", IEEE Access, vol. 5, no. June, pp. 11054-11062, 2017.

[12] J.H. Mun and H. Lim, "Cache Sharing Using Bloom Filters in Named Data Networking", J. Netw. Comput. Appl., vol. 90, pp. 74-82, 2017.

[13] A. Hidayat, F. Bukhari, and H. Sukoco, "Bloom filter implementation in cache with low level of false positive", Telkomnika (Telecommunication Comput. Electron. Control., vol. 15, no. 4, pp. 1911-1916, 2017.

[14] K. Assantachai, K. Kaneko, and F. Teraoka, "A Hybrid Cache Management Scheme for Information Centric Networking", in Institute of Electronics, Information and Communication Engineers, 2015, pp. 4-9.

[15] S. Shailendra, S. Sengottuvelan, H.K. Rath, B. Panigrahi, and A. Simha, "Performance Evaluation of Caching Policies in NDN - an ICN Architecture", in IEEE Region 10 Conference (TENCON), 2016, pp. 1117-1121.

[16] M.H. Awadalla and A. Sadek, "An efficient cache organization for on-chip multiprocessor networks", Int. J. Electr. Comput. Eng., vol. 5, no. 3, pp. 503-517, 2015.

[17] P. Charan, T. Usmani, R. Paulus, and S.H. Saeed, "A Cooperative Cache Management Scheme for IEEE802 . 15 . 4 based Wireless Sensor Networks", Int. J. Electr. Comput. Eng., vol. 8, no. 3, pp. 1701-1710, 2018.

[18] L. Rui, S. Yang, and H. Huang, "A proactive multi-level cache selection scheme to enhance consumer mobility support in Named Data Networking", Int. J. Distrib. Sens. Networks, vol. 13, no. 10, 2017.

[19] N. Abani and M. Gerla, "Proactive Caching with Mobility Prediction under Uncertainty in Information-centric Networks", in 4th ACM Conference on Information-Centric Networking (ICN 2017), 2017, pp. 88-97.

[20] M.B. Lehmann, M.P. Barcellos, and A. Mauthe, "Providing Producer Mobility Support in NDN Through Proactive Data Replication", in Network Operations and Management Symposium (NOMS 2016), 2016, no. Noms, pp. 383391.

[21] A. Azgin, R. Ravindran, and G. Wang, "Mobility Study for Named Data Networking in Wireless Access Networks", in Next-Generation Networking Symposium, 2014, pp. 3252-3257.

[22] S. Podlipnig and L.B. Osz, "A Survey of Web Cache Replacement Strategies", ACM Comput. Surv., vol. 35, no. 4, pp. 374-398, 2003. 
[23] M. Zhang, H. Luo, and H. Zhang, "A Survey of Caching Mechanisms in Information centric Networking”, IEEE Commun. Surv. TUTORIALS, vol. 17, no. 3, pp. 1473-1499, 2015.

[24] E. Hattab and S. Kawasmah, "A Survey of Replacement Policies for Mobile Web Caching", in International Conference on Developments of E-Systems Engineering, 2015.

[25] D. Saxena and I.I.T. Roorkee, "Named Data Networking: A Survey”, Comput. Sci. Rev., vol. 19, pp. 15-55, 2016.

[26] H. Situmorang, N.R. Syambas, and T. Juhana, "The Effect of Scaling the Size of Topology and Content Stored on the Named Data Networking", in International Conference on Telecommunication Systems, Services, and Applications 2016, 2016, pp. 16-21.

[27] G. Zhang, B. Tang, P. Wang, Y. Wu, and X. Zhang, "Performance Assessment of cache strategies in Content Centric Network", Inf. Technol. J., vol. 12, no. 23, pp. 7083-7089, 2013.

[28] V. Sourlas, P. Flegkas, and G.S. Paschos, "Storage Planning and Replication Assignment in Content-Centric Publish / Subscribe Networks", Int. J. Comput. Telecommun. Netw., vol. 55, no. 18, pp. 4021-4032, 2010.

[29] X. Hu, J. Gong, G. Cheng, and C. Pant, "Enhancing In-network Caching by Coupling Cache Placement, Replacement and Location”, in IEEE ICC 2015 - Next Generation Networking Symposium, 2015, pp. 5672-5678.

[30] Y. Wang, M. Xu, and Z. Feng, "Hop-based Probabilistic Caching for Information -Centric Networks", in Globecom 2013- Next Generation Networking Symposium, 2013, pp. 2102-2107.

[31] N. Chauhan and L.K. Awasthi, "Prefetching based Cooperative Caching in Mobile Adhoc Networks", in International Conference on Emerging Trends in Computer and Electronics Engineering, 2012, pp. 60-64.

[32] G. Rossini and D. Rossi, "A dive into the caching performance of Content Centric Networking", in 2012 IEEE 17th International Workshop on Computer Aided Modeling and Design of Communication Links and Networks, 2012.

[33] H. Chao, W.J. Jian, H. Cho, C. Tsai, and J. Pan, "Prediction-Based Cache Adaptation for Named Data Networking", J. Comput., vol. 27, no. 1, pp. 45-55, 2015.

[34] J. Ren et al., "MAGIC : a Distributed MAx-Gain In-network Caching Strategy in Information-Centric Networks", in 2014 IEEE INFOCOM Workshop on Name-Oriented Mobility, 2014, pp. 470-475.

[35] S. Kang, S. Lee, and Y. Ko, "A Recent Popularity Based Dynamic Cache Management for Content Centric Networking", in Fourth International Conference on Ubiquitous and Future Networks (ICUFN), 2012, pp. 219 224.

[36] W. Zhang, R. Fan, F. Liu, and P. Lai, "Energy-Aware Caching”, in IEEE 21st International Conference on Parallel and Distributed Systems, 2015, pp. 473-480.

[37] I. Psaras, W.K. Chai, and G. Pavlou, "Probabilistic In-Network Caching for Information-Centric Networks", in second edition of the ICN workshop on Information-centric networking, 2012, pp. 55-60.

[38] C. Fricker, P. Robert, J. Roberts, and N. Sbihi, "Impact of traffic mix on caching performance in a content-centric network", in Workshop on Emerging Design Choices in Name-Oriented Networking, 2012, pp. 310-315.

[39] N. Chand, R.C. Joshi, and M. Misra, "Cooperative Caching Strategy in Mobile Ad Hoc Networks Based on Clusters", Wirel. Pers. Commun., vol. 43, no. 1, pp. 41-63, 2007.

[40] M. Amadeo and A. Molinaro, "CHANET: A Content-Centric Architecture for IEEE", in 2011 International Conference on the Network of the Future, 2011, pp. 122-127.

[41] G. Carofiglio, V. Gehlen, D. Perino, and B. Labs, "Experimental Evaluation of Memory Management in ContentCentric Networking”, in IEEE International Conference on Communications (ICC), 2011, pp. 1-6.

[42] N.E. Majd, S. Misra, and R. Tourani, "Split-Cache: A Holistic Caching Framework for Improved Network Performance in Wireless Ad Hoc Networks", in Global Communications Conference (GLOBECOM), 2014, pp. $137-142$

[43] Y. Wang, "Hierarchical Cooperative Caching in Mobile Opportunistic Social Networks", in Globecom 2014 - Ad Hoc and Sensor Networking Symposium, 2014, no. 3, pp. 411-416.

[44] W. Quan, Y. Liu, X. Jiang, and J. Guan, "Intelligent popularity-aware content caching and retrieving in highway vehicular networks", EURASIP J. Wirel. Commun. Netw., 2016.

[45] Y. Kim, Y. Kim, J. Bi, and I. Yeom, "Differentiated forwarding and caching in named-data networking", J. Netw. Comput. Appl., vol. 60, pp. 155-169, 2016.

[46] W. Feng and Y. Zhang, "A General Cache Partition Model for Multiple QoS Classes", in International Conference on Computational Intelligence for Modelling, Control and Automation, and International Conference on Intelligent Agents, Web Technologies and Internet Commerce, 2005, pp. 0-5.

[47] A. Virgillito, "Publish/Subscribe Communication Systems: from Models to Applications", Universita degli Studi di Roma "La Sapienza", 2003.

[48] A. Rao, P. Kumar, and N. Chauhan, "Energy Efficient Dynamic Group Caching in Mobile Ad hoc Networks for Improving Data Accessibility", in International Conference on Recent Trends In Information Technology, 2012, pp. 372-376.

[49] C. Fang, F.R. Yu, S. Member, T. Huang, J. Liu, and Y. Liu, "A Survey of Green Information-Centric Networking: Research Issues and Challenges”, IEEE Commun. Surv. Tutorials, vol. 17, no. 3, pp. 1455-1472, 2015.

[50] A.M.E. Tamizharasi, J.J.S.M.E, A.K. Priya, and R. Maarlin, "Energy Aware Heuristic Approach for Cluster Head Selection in Wireless Sensor Networks", Bull. Electr. Eng. Informatics, vol. 6, no. 1, pp. 70-75, 2017. 


\section{BIOGRAPHIES OF AUTHORS}

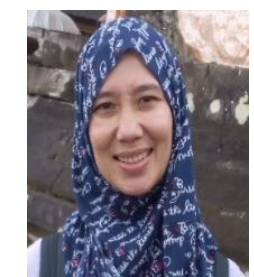

Leanna Vidya Yovita, a Ph.D. student at the School of Electrical Engineering and Informatics, Bandung Institute of Technology. She got her Bachelor and Master degree from Telkom University. Currently working as a lecturer at Telkom University, S1 Telecommunication Engineering program, teaching computer network, algorithm, and network engineering.She and the team have produced atextbook, titled "Jaringan Komputer" for the students of Telkom University. Research interests include Computer Networks, Content Centric Network, and Network Engineering.

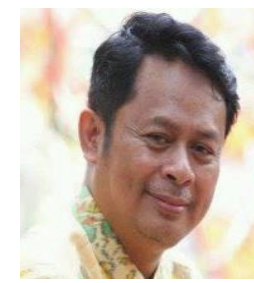

Nana Rachmana Syambas. Professor Nana Rachmana Syambas was graduated with his bachelor degree in Electrical Engineering Department, ITB in 1983. He got his Master by Research degree from Royal Melbourne Institute of Technology, Australia in 1990 and adoctoral degree from School of Electrical Engineering and Informatics, ITB in 2011. He has been a lecturer at School of Electrical Engineering and Informatics, ITB since 1984. His research interest includes Telecommunication Networks, Telematic Services, Content Centric Network (CCN), Software Defined Network (SDN), Protocol engineering and Tele-traffic engineering. He has authored or coauthored over 80 published articles. 